\title{
Acute Exacerbation of Chronic Obstructive Pulmonary Disease: Predictors of Outcome
}

\author{
Esraa A. Saad ${ }^{1}$, Kamal A. Ata ${ }^{2}$, Mona T. Hussein ${ }^{3}$, Abdellah H. Khalil ${ }^{4}$ \\ 1 Administrator ofChest Disease and Tuberculosis,Sohag University. \\ 2 Professor of Chest Disease and Tuberculosis, Sohag University. \\ 3 Assisstant Professor of Chest Disease and Tuberculosis, SohagUniversity. \\ 4 Assisstant Professor of Chest Disease and Tuberculosis, Sohag University.
}

\begin{abstract}
Background:Acute exacerbation of chronic obstructive pulmonary disease (AECOPD) is considered to be an important cause of morbidity, intensive care unit (ICU) admissionand mortality in COPD patients.

Objectives:to identify the factors which could predict the outcome ofCOPDpatients.

Patients and methods: A prospective study was conducted at Chest Department of Sohag University Hospital during the period from May 2016 to August 2017 and included 101 COPD patients with AECOPD. Patients were deemed to have AECOPD if this diagnosis appeared on their clinical histories. The studied variables included clinical parameters (symptoms \& signs), spirometry and laboratory tests (complete blood count, serum creatinine, liver function tests -ALT, AST and serum albuminserum electrolytes, arterial blood gas test and sputum cultures), radiological data(plain chest X-ray, CT chest (if indicated) and echocardiographic data for every patient. The outcome in the studyincluded improvement or non-improvement (referral to ICU or death).
\end{abstract}

Results:The study included 101 patients with AECOPD, the mean age of the patients was $60 y e a r s, 66.34 \%$ of them were males and $33.66 \%$ females, according tothe outcome; 83 cases improved, 18 cases had poor outcome (i.e. need ICU admissionordied). Bacterial growth, in the sputum culture, was recorded in $65.35 \%$ of the cases. The most frequently recorded bacterial organism were: Streptococcus pneumonia, Haemophilus influenza and Pseudomonas aeruginosa $(14.85 \%, 13.87 \%$ and $10.89 \%$ respectively). The factors, which had significant relation to poor outcome, were: male gender $(\mathrm{P}=0.04)$, frequent exacerbation $(\mathrm{P}=0.003)$, history of $\geq 2$ hospital admission and previous ICU admission in the last year $(\mathrm{P}=0.004$ and 0.003 in order), history of prior LTOT $(\mathrm{P}=0.006)$,altered consciousness, tachycardia, tachypnea, fever, flapping tremor, pedal edema $(\mathrm{P}=0.009,0.02,<0.0001$, $0.03,<0.0001$ and 0.008 in order), associated comorbidities (bronchiectasis, corpulmonale and $\mathrm{DM} ; \mathrm{P}=0.047,0.005$ and 0.008 respectively), lower mean values of $\mathrm{pH}, \mathrm{PaO}_{2}, \mathrm{SaO}_{2}$ and higher mean values of $\mathrm{PaCO}_{2}$ on admission $(\mathrm{P}=0.007,0.003$, 0.001 and 0.01 in order), leukocytosis, thrombocytopenia, elevated serum creatinine, elevated liver enzymes and hypoalbuminemia $(\mathrm{P}=0.008,0.001,0.02,0.001$ and 0.007 in order), presence of cardiomegaly or bronchiectaic changes as radiological findings $(\mathrm{P}=0.001$ and 0.047 in order $)$, severe pulmonary artery hypertension as an echocardiographic finding $(\mathrm{P}=0.03)$, lower mean values of $\mathrm{FEV} 1$ and $\mathrm{FVC}(\mathrm{P}=0.01$ and 0.02 in order), Staph. aureusand $\mathrm{P}$. aeruginosa isolation in sputum cultures $(\mathrm{P}<0.0001$ and 0.002 in order).

Conclusion:The significant factors in predicting poor outcome of AECOPD were: male gender, frequent exacerbations, prior hospital ( $\geq 2$ hospital admission/year) and ICU admission in the last year, history of prior LTOT, associated comorbidities (bronchiectasis,corpulmonale and DM), consciousness alteration, tachycardia, tachypnea, fever, flapping tremor, lower limb edema, arterial blood gas parameters on 
admission (higher mean values of $\mathrm{PaCO}_{2}$ and lower mean values of $\mathrm{pH}, \mathrm{PaO}_{2}$ and $\mathrm{SaO}_{2}$ ), leukocytosis, thrombocytopenia, elevated serum creatinine, higher mean levels of ALT and AST, hypoalbuminemia, presence of cardiomegaly or bronchiectatic changes as radiological findings, severe pulmonary artery hypertension as an echocardiographic finding, lower mean values of $\mathrm{FEV}_{1}$ and FVC, P.aeruginosa and Staph.aureus isolation in sputum cultures.

Key words:AECOPD, COPD, predictors, outcome.

\section{Introduction}

The Global Initiative for Obstructive Lung Disease (GOLD) defined an AECOPD as an acute worsening of respiratory symptoms that result in additional therapy(GOLD, 2018).

It is important to identify the prognostic factors of it as a respect to the fact that AECOPD is an important and common cause of emergency room visits and is a major cause of morbidity and death and the fact that following an acute exacerbation, many patients experience a transient or permanent decrease in the quality of life (Connor et al., 1996 \&Seemungal et al., 2000), thus the economic and social burden of COPD exacerbation are extremely high (Lopez et al. 2006).

The aim of the study: We aimedto identify thefactors which could predict the outcome of acute exacerbation in COPD patients.

\section{Patients and methods:This} prospective study was conducted in the Chest Department of Sohag University Hospital. The study included 101 patients with AECOPD who were admitted to the department from outpatient chest clinic and emergency department during the period from May 2016 to August 2017. The study was approved by the medical ethics committee of Sohag Faculty of Medicine. Consent was taken from every patient to participate in the study.Patients who were $<40$ years old, critical patients who needed ICU admission at the time of first evaluation in emergency room, and the patients who had pulmonary tuberculosis or acute coronary syndrome were excluded from the study.

Acute exacerbation of COPD was diagnosed using the following symptoms (Anthonisen et al., 1987):recent rapid worsening of dyspnea, presence of sputum purulence and increase in sputum volume.According to these symptoms, exacerbations were classified into (Anthonisen et al., 1987):

- Type 1 (severe AECOPD): exacerbations include increased dyspnea, sputum volume and sputum purulence.

- Type 2 (moderate AECOPD): exacerbations involve any two of those symptoms.

- Type 3 (mild AECOPD): exacerbations includeonly one of those symptoms plus one of the following (an upper respiratory tract infection in the past 5 days, fever without other causes, increased wheezing or cough, or an increase in heart rate or respiratory rate by $20 \%$ compared with the baseline readings).

Patients who had positive sputum culture, fever or purulent sputum, were considered to have infectious AECOPD, while the others who had none of them were considered to have non-infectious AECOPD (Elkorashy et al., 2014).

All patients were subjected to full clinical evaluation (history and examination), plain chest $\mathrm{x}$ ray,electrocardiogram, computed tomography (if indicated), abdominal ultrasonography, echocardiography, 
SOHAG MEDICAL JOURNAL

Vol. 23 No.1 Jan 2019

complete blood count and arterial blood gases analysis on admission $(\mathrm{pH}$, $\left.\mathrm{PaCO}_{2}, \quad \mathrm{PaO}_{2}, \quad \mathrm{SaO}_{2} \% \quad \& \mathrm{HCO}_{3}\right)$ the samples were analyzed using automated blood gases analyzer (ABL800 FLEX blood gas analyzer, radiometer, USA), blood chemistrywas done, including serum creatinine, liver enzymes (ALT\&AST), serum albumin and serum electrolytes $\left(\mathrm{Na}^{+}, \mathrm{K}^{+}, \mathrm{C} \mathrm{a}^{++}\right)$ (Roche/Hitachi cobas c 311systen, Germany).

\section{Sputum examination:}

Bacterial sputum cultures was done for every patient. At hospital admission, the sputum was collected by spontaneous or induced expectoration (using nebulized hypertonic saline). Early morning sputum samples were collected in sterile containers before starting antibiotic treatment at the hospital. The samples were transferred to the bacteriology laboratory of Sohag University Hospital where they were examined and the results were reported by specialists. The sputa were cultured only if the quality criteria were met in a sputum Gram stain $(<10$ epithelial cells and >25polymorphonuclear leukocytes per low magnification field $\mathrm{x} 100$ ). Samples were cultured on sheep blood agar plates and mcConkey agar (Dylan Inc., Edomonton, Alberta), then incubated at $37^{\circ} \mathrm{C}$ in a moist atmosphere containing $5 \% \mathrm{CO}_{2}\left(\mathrm{CO}_{2}\right.$ incubator, Grant Instruments Ltd) for 24hours (if no results further incubation until $48 \mathrm{hr}$ was done). Only isolates with higher than 105 colony forming units were reported as a positive growth(Murray et al., 2003).VITEK 2 compact automated microbial system (bioMérieux, Inc. Hazelwood, USA) was used for identification of the bacterial microorganisms using colorimetric reagent cards that are incubated and interpreted automatically.

Pulmonary function tests (PFT):
Acute Exacerbation of Chronic Obstructive

Esraa A. Saad.et al

Pulmonary function tests data were obtained from the previous records of the patients and when these were not available, PFTs were done while the patients were stable and free from all the symptoms and the signs of acute exacerbation (at least after two weeks). The data were obtained for 91 patients, as previous records and follow up data for the others were not available. PFTs were performed with a spirometer of computer processing (Jaeger Master Screen Diffusion, Viasys Healthcare, Gmbh, Hoechberg, Germany). Postbronchodilator spirometry measurements were recorded for the studied population to measure the values of forced expiratory volume in $1^{\text {st }}$ second $\left(\mathrm{FEV}_{1}\right)$, forced vital capacity (FVC) and $\mathrm{FEV}_{1} / \mathrm{FVC}$ ratio. Postbronchodilator values were used for the evaluation of COPD severity, according to GOLD guidelines (Stage I: mild COPD, $\mathrm{FEV}_{1}>80 \%$ of predicted $\mathrm{FEV}_{1}$ - Stage II: moderate COPD, 50\% $\leq \mathrm{FEV}_{1}<80 \%$-Stage III: severe COPD, $30 \% \leq \mathrm{FEV}_{1}<50 \%$-Stage IV: very severe COPD, $\mathrm{FEV}_{1}<30 \%$ ) (GOLD, 2018).

Outcome in the studied population was assessed as the following (Chow et al., 1992 \&Mantero et al., 2017):

- Improvement was considered if patients continued their treatment in the ward until resolution or reduction in the symptoms and signs occurred without appearance of new symptoms or signs associated with the exacerbation.

- Non improvement was considered when worsening of symptoms or signs occurred and led toreferral to intensive care unit (ICU) or death.

Statistical analysis: Data was analyzed using STATA intercooled version 14.2. Quantitative data was represented as mean, standard deviation. Data was analyzed using student t-test to compare means of two groups. When the data was not 
SOHAG MEDICAL JOURNAL

Vol. 23 No.1 Jan 2019

normally distributed Mann-Whitney

test was used. Qualitative data was

presented as number and percentage and compared using either Chi square
Acute Exacerbation of Chronic Obstructive

Esraa A. Saad.et al

test or fisher exact test. Odds ratios were obtained from logistic regression analysis. $\mathrm{P}$ value was considered significant if it was less than 0.05 .

\section{Results}

The study included 101 patient with AECOPD with mean age of 60 years, $66.34 \%$ of the cases were males and $33.66 \%$ of the cases were females. $47.52 \%$ of the cases were ex-smokers, $31.68 \%$ of the cases were current smokers and $20.79 \%$ of the cases were non-smokers(Table 1).

\begin{tabular}{|c|c|}
\hline Variable & $\begin{array}{l}\text { Summary } \\
\text { statistics }\end{array}$ \\
\hline Age (year) $[$ mean $\pm \mathrm{SD}($ range $)]$ & $60 \pm 8.52(44-85)$ \\
\hline $\begin{array}{l}\text { Gender }(\mathrm{n}, \%) \quad \text { Females } \\
\text { Males }\end{array}$ & $\begin{array}{l}34(33.66 \%) \\
67(66.34 \%)\end{array}$ \\
\hline $\begin{array}{r}\text { Smoking status }(\mathrm{n}, \%) \text { Non-smoker } \\
\text { Current } \\
\text { Ex-smoker }\end{array}$ & $\begin{array}{l}21(20.79 \%) \\
32(31.68 \%) \\
48(47.52 \%)\end{array}$ \\
\hline
\end{tabular}

Table (1): Demographic characteristics of the studied population $(n=101)$

\begin{tabular}{|l|c|}
\hline Etiology* $^{*}$ & Number \% \\
\hline $\begin{array}{l}\text { Infectious } \\
\text { Positive bacterial sputum culture } \\
\text { Negative bacterial sputum culture } \\
\text { Total }\end{array}$ & $66(65.35 \%)$ \\
$29(28.71 \%)$ \\
$95(94.06 \%)$ \\
\hline Non infectious & $6(5.94 \%)$ \\
\hline
\end{tabular}

Table(2): Etiology of AECOPD of the studied population $(n=101)$

*Patients who had positive sputum culture, fever or purulent sputum, were considered to have infectious AECOPD, while the others who had none of them were considered to have noninfectious AECOPD (Elkorashy et al., 2014).

Table (2)shows that infectious causes of AECOPD weresuspected in94.06\% of the patients. Positive bacterial sputum culture was found in $65.35 \%$ of all cases. 


\begin{tabular}{|l|l|}
\hline Microbiology & Number (\%) \\
\hline No growth & $31(30.65 \%)$ \\
\hline Positive sputum culture & $66(65.35 \%)$ \\
- Streptococcus pneumonia & $15(14.85 \%)$ \\
- Haemophilus influenza & $14(13.87 \%)$ \\
- Pseudomonas aeruginosa & $11(10.89 \%)$ \\
- Staphylococcus aureus & $9(8.9 \%)$ \\
- Klebsiella pneumonia & $6(5.94 \%)$ \\
- Streptococcus pyogenes & $5(4.96 \%)$ \\
- Streptococcus parasanguinis & $4(3.96 \%)$ \\
Enterococci & $2(1.98 \%)$ \\
\hline
\end{tabular}

Table (3): Microbiological findings (by sputum culture) in the studied population $(n=101)$

Table (3) shows that $65.35 \%$ of the patients had positive sputum cultures. The most frequent bacterial growth were: Strept.pneumonia, H. influenza, P. aeruginosa and Staph.aureus $(14.85 \%, 13.87 \%, 10.89 \%$ and $8.9 \%$ respectively).

\begin{tabular}{|l|l|}
\hline Outcome & Number (\%) \\
\hline Improvement & $83(82.18 \%)$ \\
\hline $\begin{array}{l}\text { Non improvementICU } \\
\text { admissionDeath }\end{array}$ & $14(13.86 \%)$ \\
Total & $18(17.86 \%)$ \\
\hline
\end{tabular}

Table(4): Outcome of the studied population $(n=101)$

ICU: intensive care unit

Table (4) shows that $82.18 \%$ of the cases improved with management in the ward of Chest Department, $13.86 \%$ of the cases were referred to ICU, and 3.96\% of the cases died.

\begin{tabular}{|l|c|c|c|c|}
\hline \multicolumn{1}{|c|}{ Variable } & Improved(n=83) & $\begin{array}{c}\text { Not } \\
\text { improved(n=18) }\end{array}$ & $\begin{array}{c}\text { Odds ratio } \\
(95 \% \text { CI })\end{array}$ & $\begin{array}{c}\text { P } \\
\text { value }\end{array}$ \\
\hline Age (year) (mean \pm SD) & $59.84 \pm 8.04$ & $62.72 \pm 10.34$ & $1.04(0.98-1.10)$ & 0.2 \\
\hline $\begin{array}{l}\text { Gender(n,\%) } \\
\text { Females } \\
\text { Males }\end{array}$ & $32(38.55 \%)$ & $2(11.11 \%)$ & 1 & \\
\hline Smoking status (n,\%) & $\mathbf{5 1}(\mathbf{6 1 . 4 5 \% )}$ & $\mathbf{1 6 ( 8 8 . 8 9 \% )}$ & $\mathbf{5 . 0 1}(\mathbf{1 . 0 8 - 2 3 . 2 9 )})$ & $\mathbf{0 . 0 4}$ \\
$\begin{array}{l}\text { Smokers } \\
\text { Nonsmokers }\end{array}$ & $64(77.1 \%)$ & $6(88.89 \%)$ & $2.38(0.50-11.26)$ & 0.28 \\
& $19(22.9 \%)$ & $2(11 . .11 \%)$ & 1 & \\
\hline
\end{tabular}

Table(5): Relation between patient 's outcome and demographic characteristics

Table (5) shows that poor outcome was significantly related to male gender $(\mathrm{P}=$ 0.04). Patients with poor outcome had higher mean age in comparison with patients who improved, but this relation was statistically not significant. 
SOHAG MEDICAL JOURNAL

Vol. 23 No.1 Jan 2019

\begin{tabular}{|c|c|c|c|c|}
\hline I. 23 No.1 Jan 2019 & & & a A. Saad.et a & \\
\hline Variable & $\begin{array}{c}\text { Improved } \\
(\mathrm{n}=83)\end{array}$ & $\begin{array}{c}\text { Not improved } \\
(\mathrm{n}=18)\end{array}$ & $\begin{array}{l}\text { Odds ratio }(95 \% \\
\text { CI) }\end{array}$ & $\begin{array}{l}P \\
\text { value }\end{array}$ \\
\hline $\begin{array}{l}\text { Duration of the disease } \\
\text { (year) }(\text { mean } \pm \mathrm{SD})\end{array}$ & $11.88 \pm 8.46$ & $13.83 \pm 6.93$ & $1.03(0.97-1.09)$ & 0.36 \\
\hline Prior LTOT $(\mathrm{n}, \%)$ & $12(14.46 \%)$ & $8(44.44 \%)$ & $4.73(1.56-14.4)$ & 0.006 \\
\hline $\begin{array}{l}\text { Prior ICU admission ( last } \\
\text { year)(n, } \%)\end{array}$ & $5(6.02 \%)$ & $6(33.33 \%)$ & $7.8(2.01-29.59)$ & 0.003 \\
\hline $\begin{array}{l}\text { Prior hospitalization (last } \\
\text { year)(n,\%) } \\
0 \\
1 \\
\quad \geq \mathbf{2}\end{array}$ & $\begin{array}{c}39(46.99 \%) \\
23(27.71 \%) \\
\mathbf{2 1}(\mathbf{2 5 . 3 \%})\end{array}$ & $\begin{array}{l}2(11.11 \%) \\
5(27.78 \%) \\
\mathbf{1 1}(\mathbf{6 1 . 1 1 \% )}\end{array}$ & $\begin{array}{c}1 \\
4.24(0.76-23.65) \\
\mathbf{1 0 . 2 1}(\mathbf{2 . 0 7 - 5 0 . 5})\end{array}$ & $\begin{array}{c}0.1 \\
\mathbf{0 . 0 0 4}\end{array}$ \\
\hline $\begin{array}{l}\text { Frequency of AECOPD( } \\
\text { last year) (mean } \pm \text { SD) }\end{array}$ & $2.25 \pm 1.17$ & $3.22 \pm 1$ & $2.15(1.29-3.6)$ & 0.003 \\
\hline $\begin{array}{c}\text { Exacerbation severity } \\
\text { (n,\%) Severe } \\
\text { Moderate/ Mild }\end{array}$ & $\begin{array}{l}60(72.29 \%) \\
23(27.71 \%)\end{array}$ & $\begin{array}{l}17(94.44 \%) \\
1(5.56 \%)\end{array}$ & $\begin{array}{l}6.52(0.82-51.81) \\
1\end{array}$ & 0.08 \\
\hline
\end{tabular}

LTOT: long term oxygen therapy.

Acute Exacerbation of Chronic Obstructive Esraa A. Saad.et al

Table (6): Relation between the patient's outcome and the characteristics of the disease and current exacerbation

Table(6) shows that there were significant relations between poor outcome andhistory of prior LTOT, priorICU admission,previous $\geq 2$ hospital admissions in the last year and higher mean frequency of exacerbation in the last year $(\mathrm{P}=0.006,0.003,0.004$ and 0.003 in order).

\begin{tabular}{|c|c|c|c|c|}
\hline Variable & $\underset{(\mathrm{n}=83)}{\text { Improved }}$ & $\begin{array}{c}\text { Not } \\
\text { improved } \\
(n=18)\end{array}$ & $\begin{array}{l}\text { Odds ratio } \\
\text { (95\% CI) }\end{array}$ & $P$ value \\
\hline $\begin{array}{l}\text { Increased sputum } \\
\text { volume }(\mathrm{n}, \%)\end{array}$ & $76(91.57 \%)$ & $18(100 \%)$ & $3.83(0.00)$ & 0.99 \\
\hline Increased dyspnea(n,\%) & $82(98.76 \%)$ & $18(100 \%)$ & $3.55(0.00)$ & 1 \\
\hline $\begin{array}{l}\text { Altered consciousness } \\
(\mathrm{n}, \%)\end{array}$ & $10(12.05 \%)$ & $7(38.89 \%)$ & $4.65(1.46-14.8)$ & 0.009 \\
\hline Cyanosis (n,\%) & $48(57.83 \%)$ & $16(88.9 \%)$ & $3.47(0.93-12.92)$ & 0.06 \\
\hline Pulse rate $($ mean \pm SD) & $100.77 \pm 15.6$ & $111.0 \pm 20.1$ & $1.04(1.01-1.07)$ & 0.02 \\
\hline $\begin{array}{l}\text { Respiratory rate (mean } \\
\pm \mathrm{SD} \text { ) }\end{array}$ & $26.2 \pm 3.99$ & $31.11 \pm 3.99$ & $1.36(1.16-1.61)$ & $<0.0001$ \\
\hline Fever $(\mathrm{n} \%)$ & $51(61.45 \%)$ & $17(94.44 \%)$ & 10.67(1.35-84.08) & $\mathbf{0 . 0 3}$ \\
\hline Flapping tremor $(\mathrm{n}, \%)$ & $18(31.67 \%)$ & $14(77.8 \%)$ & $12.64(3.7-43.14)$ & $<0.0001$ \\
\hline Pedal edema $(\mathrm{n}, \%)$ & $33(39.76 \%)$ & $16(88,9 \%)$ & $5.04(1.53-16.65)$ & 0.008 \\
\hline
\end{tabular}

Table (7):Relation between patient's outcome and their clinical data 
SOHAG MEDICAL JOURNAL

Vol. 23 No.1 Jan 2019
Acute Exacerbation of Chronic Obstructive

Esraa A. Saad.et al

Table (7) demonstrates that poor outcome was significantly related to presence of consciousness alteration, higher rates of pulse and respiration, fever,flapping tremorsand pedal edema, in comparison with good outcome $(\mathrm{P}=0.009,0.02,<0.0001$, $0.03,<0.0001$ and 0.008 respectively).

\begin{tabular}{|c|c|c|c|c|}
\hline Variable & $\begin{array}{c}\text { Improved } \\
(n=83)\end{array}$ & $\begin{array}{c}\text { Not improved } \\
(n=18)\end{array}$ & $\begin{array}{c}\text { Odds ratio(95\% } \\
\text { CI })\end{array}$ & P value \\
\hline Bronchiectasis (n,\%) & $8(9.64 \%)$ & $5(27.78 \%)$ & $3.60(1.02-12.8)$ & 0.047 \\
\hline Pneumonia (n,\%) & $13(15.66 \%)$ & $6(33.33 \%)$ & $2.69(0.86-8.46)$ & 0.09 \\
\hline Sleep disorders $(\mathrm{n}, \%)$ & $8(9.64 \%)$ & $3(16.67 \%)$ & $1.88(0.45-7.89)$ & 0.39 \\
\hline $\operatorname{DCP}(\mathbf{n}, \%)$ & $36(43.37 \%)$ & $15(83.33 \%)$ & $6.53(1.76-24.28)$ & 0.005 \\
\hline IHD (n,\%) & $18(21.69 \%)$ & $7(38.89 \%)$ & $2.3(0.78-9.78)$ & 0.13 \\
\hline Hypertension $(\mathrm{n}, \%)$ & $35(42.17 \%)$ & $8(44.44 \%)$ & $1.1(0.39-3.06)$ & 0.86 \\
\hline DM $(n, \%)$ & $20(24.1 \%)$ & $10(55.56 \%)$ & $3.94(1.36-11.3)$ & 0.008 \\
\hline Renal diseases $(\mathrm{n}, \%)$ & $4(4.82 \%)$ & $3(16.67 \%)$ & $3.95(0.8-19.5)$ & 0.09 \\
\hline Hepatic diseases $(\mathrm{n}, \%)$ & $7(8.43 \%)$ & $4(22.22 \%)$ & $3.1(0.8-12)$ & 0.1 \\
\hline $\begin{array}{l}\text { Comorbidity }(\mathrm{n}, \%) \\
\quad<2 \\
\geq 2\end{array}$ & $\begin{array}{l}19(22.9 \%) \\
64(77.1 \%)\end{array}$ & $\begin{array}{l}0 \\
18(100 \%)\end{array}$ & $\begin{array}{l}\text { Omitted } \\
4.54(0.00)\end{array}$ & 1 \\
\hline
\end{tabular}

DCP: decompensated corpulmonaleIHD: ischemic heart disease

DM: diabetes mellitus

\section{Table (8)Relation between patient's outcome and comorbidities}

Table (8)demonstrates that the patients with poor outcome had higher frequencies of associated comorbidities (i.e. bronchiectasis,DCP and DM) in comparison with the patients with good outcome $(\mathrm{P}=0.047,0.005$ and 0.008 respectively).

\begin{tabular}{|c|c|c|c|c|}
\hline Variable & $\begin{array}{c}\text { Improved } \\
(\mathrm{n}=83)\end{array}$ & $\begin{array}{l}\text { Not improved } \\
\quad(n=18)\end{array}$ & $\begin{array}{c}\text { Odds } \\
\text { ratio(95\% CI) }\end{array}$ & $P$ value \\
\hline $\mathbf{p H}($ mean $\pm \mathrm{SD})$ & $7.4 \pm 0.07$ & $7.35 \pm 0.06$ & $0.0001(0-0.2)$ & 0.007 \\
\hline $\begin{array}{l}\mathrm{PaCo}_{2}(\text { mean } \pm \\
\mathrm{SD})\end{array}$ & $47.73 \pm 16.83$ & $59.89 \pm 17.45$ & $1.04(1.00-1.08)$ & 0.01 \\
\hline $\mathbf{P a O}_{2}($ mean \pm SD $)$ & $56.1 \pm 16.32$ & $42.28 \pm 12.82$ & $0.93(0.88-0.98)$ & 0.003 \\
\hline $\begin{array}{ll}\mathrm{SaO}_{2} & (\text { mean } \pm \\
\mathrm{SD}) & \end{array}$ & $82.52 \pm 11.73$ & $70.64 \pm 12.78$ & $0.93(0.89-0.97)$ & 0.001 \\
\hline $\begin{array}{l}\mathrm{HCO}_{3} \cdot(\text { mean } \pm \\
\mathrm{SD})\end{array}$ & $26.36 \pm 5.44$ & $28.56 \pm 6.11$ & $1.07(0.98-1.17)$ & 0.14 \\
\hline
\end{tabular}

pH:potential of hydrogen.PaCO$:$ partial arterial tension of carbon dioxide. $\mathbf{P a O}_{2}$ : partial arterial tension of oxygen. $\mathrm{SaO}_{2}$ : arterial oxygen saturation. $\mathrm{HCO}_{3}$ : bicarbonate.

Table (9): Relation between the patient's outcome and arterial blood gas parameters on admission 
SOHAG MEDICAL JOURNAL

Vol. 23 No.1 Jan 2019
Acute Exacerbation of Chronic Obstructive

Esraa A. Saad.et al

Table(9)shows that,on admission, the patients with poor outcome had lower mean values of $\mathrm{pH}, \mathrm{PaO}_{2}$ and $\mathrm{SaO}_{2} \%$ in comparison with the patients with good outcome $(\mathrm{P}=0.007,0.003$ and 0.001 respectively). The patients with poor outcome had higher values of $\mathrm{PaCO}_{2}$ in comparison with patients with good outcome $(\mathrm{P}=0.01)$.

\begin{tabular}{|c|c|c|c|c|}
\hline Variable & Improved $(\mathrm{n}=83)$ & $\begin{array}{l}\text { Not improved } \\
\quad(n=18)\end{array}$ & $\begin{array}{c}\text { Odds } \\
\text { ratio(95\% CI })\end{array}$ & $P$ value \\
\hline Leukocytosis $(\mathrm{n}, \%)$ & $32(38.55 \%)$ & $15(83.33 \%)$ & $1.13(1.03-1.24)$ & 0.008 \\
\hline Polycythemia $(\mathrm{n}, \%)$ & $12(14.46 \%)$ & $3(16.67 \%)$ & $1.18(0.3-4.7)$ & 0.81 \\
\hline Thrombocytopenia $(\mathrm{n}, \%)$ & $7(8.54 \%)$ & $8(44.44 \%)$ & $8.57(2.56-28.7)$ & 0.001 \\
\hline $\begin{array}{l}\text { Elevated serum creatinine } \\
(\mathrm{n}, \%)\end{array}$ & $19(22.89 \%)$ & $9(50 \%)$ & $3.37(1.17-9.68)$ & $\mathbf{0 . 0 2}$ \\
\hline Elevated liver enzyme (n,\%) & $16(19.28 \%)$ & $11(61.11 \%)$ & $6.58(2.2-19.63)$ & 0.001 \\
\hline Serum albumin (mean \pm SD) & $3.62 \pm 0.58$ & $3.19 \pm 0.57$ & $0.19(0.06-0.63)$ & 0.007 \\
\hline Sodium(mean \pm SD) & $131.93 \pm 6.41$ & $128.4 \pm 8.33$ & $0.92(0.84-1.01)$ & 0.08 \\
\hline Potassium (mean \pm SD) & $3.25 \pm 0.65$ & $3.2 \pm 0.65$ & $0.88(0.36-2.12)$ & 0.78 \\
\hline Calcium (mean $\pm \mathrm{SD}$ ) & $1.01 \pm 0.08$ & $0.99 \pm 0.10$ & $\begin{array}{l}\text { 0.15 (0.0003- } \\
84.5)\end{array}$ & 0.55 \\
\hline
\end{tabular}

Table (10): Relation between the patient's outcome and the laboratory investigations

Table(10) shows that poor outcomewas significantly related to leukocytosis, thrombocytopenia, elevation of the serum level of creatinine, liver enzymes( ALT\&AST $)$ and lower meanserum level of albumin $(\mathrm{P}=0.008,0.001,0.02,0.001$ and 0.007 respectively). 
SOHAG MEDICAL JOURNAL

Vol. 23 No.1 Jan 2019

\begin{tabular}{|l|l|l|l|l|}
\hline Variable & \multicolumn{1}{|c|}{$\begin{array}{c}\text { Improved } \\
(\mathrm{n}=83)\end{array}$} & $\begin{array}{c}\text { Not improved } \\
(\mathrm{n}=18)\end{array}$ & $\begin{array}{c}\text { Odds ratio (95\% } \\
\mathrm{CI})\end{array}$ & P value \\
\hline Hyperinflation (n,\%) & $78(93.98 \%)$ & $17(94.44 \%)$ & $1.09(0.12-9.94)$ & 1 \\
\hline Cardiomegaly (n,\%) & $\mathbf{2 9}(\mathbf{3 4 . 9 4 \% )}$ & $\mathbf{1 5}(\mathbf{8 3 . 3 3 \% )}$ & $\mathbf{9 . 3 1}(\mathbf{2 . 4 9 - 3 4 . 8 2 )}$ & $\mathbf{0 . 0 0 1}$ \\
\hline $\begin{array}{l}\text { Bronchiectatic change } \\
(\mathrm{n}, \%)\end{array}$ & $\mathbf{8}(\mathbf{9 . 6 4 \%})$ & $\mathbf{5 ( 2 7 . 7 8 \% )}$ & $\mathbf{3 . 6 0}(\mathbf{1 . 0 2 - 1 2 . 8 )}$ & $\mathbf{0 . 0 4 7}$ \\
\hline Pneumothorax (n,\%) & $4(4.82 \%)$ & 0 & Omitted & \\
\hline Lung infiltrates (n,\%) & $13(15.66 \%)$ & $6(33.33 \%)$ & $2.69(0.86-8.46)$ & 0.10 \\
\hline $\begin{array}{l}\text { Pleural effusion (n,\%) } \\
\text { Hydro- } \\
\text { pneumothorax(n,\%) }\end{array}$ & 0 & $2(11.11 \%)$ & $1.60(0.30-8.68)$ & 0.63 \\
\hline \begin{tabular}{l} 
Lung abscess (n,\%) \\
\hline
\end{tabular} & 0 & $2(11.11 \%)$ & Omitted & \\
\hline
\end{tabular}

Table (11): Relation between patient's outcome and radiological findings

Table (11) shows that the frequency of cardiomegaly and bronchiectatic changes as radiological findings in the patient with poor outcome was significantly higher than that in patients with good outcome $(\mathrm{P}=0.001$ and 0.047 respectively).

\begin{tabular}{|c|c|c|c|c|}
\hline $\operatorname{PASP}(\mathbf{n}, \%)$ & Improved $(\mathrm{n}=83)$ & $\begin{array}{c}\text { Not improved } \\
(\mathrm{n}=18)\end{array}$ & $\begin{array}{l}\text { Odds } \\
\text { ratio }(95 \% \mathrm{CI})\end{array}$ & $P$ value \\
\hline $\begin{array}{ll}\text { Normal } & (<25) \mathrm{mmHg} \\
\text { Mild } & (25: 40) \mathrm{mmHg} \\
\text { Moderate } & \\
(40: 55) \mathrm{mm} H g \text { Severe } \\
(>55) \mathrm{mmHg}\end{array}$ & $\begin{array}{ll}25(30.12 \%) & 23 \\
(27.71 \%) & \\
23(27.71 \%) & \mathbf{1 2} \\
(\mathbf{1 4 . 4 6 \% )} & \end{array}$ & $\begin{array}{l}1(5.56 \%) 3 \\
(16.67 \%) \\
8(44.44 \%) \\
\quad \mathbf{6 ( 3 3 . 3 3 \% )}\end{array}$ & $\begin{array}{l}1 \\
\quad 3.26(0.32- \\
33.61) 8.70(1.0- \\
74.99) \\
\mathbf{1 2 . 5 ( 1 . 3 5 -} \\
\mathbf{1 1 5 . 7 9 )}\end{array}$ & $\begin{array}{c}0.32 \\
0.05 \\
\mathbf{0 . 0 3}\end{array}$ \\
\hline
\end{tabular}

PASP: pulmonary artery systolic pressure.

Table (12): Relation between pulmonary artery systolic pressure (according to echocardiography) and patient's outcome

Table(12) shows that poor outcome had a significant relation to severe pulmonary hypertension $(\mathrm{P}=0.03)$. 
SOHAG MEDICAL JOURNAL

Vol. 23 No.1 Jan 2019
Acute Exacerbation of Chronic Obstructive Esraa A. Saad.et al

\begin{tabular}{|c|c|c|c|c|}
\hline$\overline{\text { Variable }}$ & Improved $(\mathrm{n}=82)$ & $\begin{array}{c}\text { Not } \\
\operatorname{improved}(n=9)\end{array}$ & $\begin{array}{c}\text { Odds } \\
\text { ratio(95\% CI })\end{array}$ & $P$ value \\
\hline $\mathbf{F E V} \mathbf{V}_{\mathbf{1}}(\mathrm{L})($ mean $\pm \mathrm{SD})$ & $1.07 \pm 0.46$ & $0.58 \pm 0.2$ & $0.003(0.00-0.3)$ & 0.01 \\
\hline $\mathbf{F V C}(\mathrm{L})($ mean $\pm \mathrm{SD})$ & $1.86 \pm 0.74$ & $1.19 \pm 0.34$ & $\begin{array}{l}0.14(0.03-0.69) \\
\end{array}$ & 0.02 \\
\hline $\begin{array}{l}\mathrm{FEV}_{1} / \mathrm{FVC} \% \quad(\text { mean } \pm \\
\text { SD) }\end{array}$ & $57.24 \pm 9.37$ & $51.82 \pm 17.94$ & $\begin{array}{l}0.59(0.89- \\
1.02)\end{array}$ & 0.15 \\
\hline $\begin{array}{l}\text { COPD staging }(\mathrm{n}, \%) \text { II/ } \\
\text { III IV }\end{array}$ & $\begin{array}{l}55(67.07 \%) \\
\mathbf{2 7}(\mathbf{3 2 . 9 3 \% )}\end{array}$ & $\begin{array}{l}2(22.22 \%) \\
\mathbf{7}(\mathbf{7 7 . 7 8 \%})\end{array}$ & $\begin{array}{l}1 \\
7.13(1.38- \\
36.66)\end{array}$ & 0.02 \\
\hline
\end{tabular}

*Spirometric parameters were recorded foronly 91 patient so the total number of the studied population in this table is 91 .

$\mathbf{F E V}_{1}$ : forced expiratory volume in $1^{\text {st }}$ secondFVC: forced vital capacity

Table (13) shows that the patients with poor outcome had significantly lower mean values of $\mathrm{FEV}_{1}$ and $\mathrm{FVC}(\mathrm{P}=0.01$ and 0.02 respectively) in comparison with the patient who improved. Poor outcome had a significant relationship with severe COPD stage (stage IV) $(\mathrm{P}=0.02)$.

\begin{tabular}{|l|c|c|c|c|}
\hline Etiology & $\begin{array}{c}\text { Improved } \\
(\mathrm{n}=83)\end{array}$ & $\begin{array}{c}\text { Not improved } \\
(\mathrm{n}=18)\end{array}$ & $\begin{array}{c}\text { Odds ratio(95\% } \\
\text { CI) }\end{array}$ & P value \\
\hline Infectious (n,\%) . . & $51(61.5 \%)$ & $\begin{array}{c}15(83.3 \%) \\
3(16.7 \%) \\
\text { Positive sputum culture }\end{array}$ & $\begin{array}{c}3.14(0.84-11.7) \\
0.44(0.12-\end{array}$ & 0.090 .22 \\
$\begin{array}{l}\text { Negative sputum } \\
\text { cultureNoninfectious } \\
(\mathrm{n}, \%)\end{array}$ & $\begin{array}{c}26(31.3 \%) \\
6(7.2 \%)\end{array}$ & 0 & $1.65)$ Omitted & \\
\hline
\end{tabular}

Table (14): Relation between the etiology of AECOPD and patient's outcome

As regard the etiology of AECOPD, table (14) shows that bacterial infection was more frequent among the patients who had poor prognosis but statistically insignificant $(\mathrm{P}=0.09)$. 
SOHAG MEDICAL JOURNAL

Vol. 23 No.1 Jan 2019
Acute Exacerbation of Chronic Obstructive Esraa A. Saad.et al

\begin{tabular}{|c|c|c|c|c|}
\hline Bacterial growth & $\begin{array}{l}\text { Improved } \\
(\mathrm{n}=83)\end{array}$ & $\begin{array}{c}\text { Not } \\
\text { improved } \\
(\mathrm{n}=18)\end{array}$ & $\begin{array}{c}\text { Odds } \\
\text { ratio(95\% CI) }\end{array}$ & $P$ value \\
\hline $\begin{array}{l}\text { Streptococcus } \\
\text { pneumonia }\end{array}$ & $15(18.07 \%)$ & 0 & Omitted & \\
\hline Haemophilus influenza & $12(14.46 \%)$ & $2(11.11 \%)$ & $0.53(0.08-3.7)$ & 0.51 \\
\hline $\begin{array}{l}\text { Pseudomonas } \\
\text { aeruginosa }\end{array}$ & $5(6.02 \%)$ & $6(33.33 \%)$ & $0.07(0.01-0.39)$ & 0.002 \\
\hline $\begin{array}{l}\text { Staphylococcus } \\
\text { aureus }\end{array}$ & $2(2.41 \%)$ & $7(38.89 \%)$ & $\begin{array}{l}0.03(0.004- \\
0.18)\end{array}$ & $<0.0001$ \\
\hline Klebsiella pneumonia & $6(7.23 \%)$ & 0 & Omitted & \\
\hline $\begin{array}{l}\text { Streptococcus } \\
\text { pyogenes }\end{array}$ & $5(6.02 \%)$ & 0 & Omitted & \\
\hline $\begin{array}{l}\text { Streptococcus } \\
\text { parasanguinis }\end{array}$ & $4(4.82 \%)$ & 0 & Omitted & \\
\hline Enterococci & $2(2.41 \%)$ & 0 & Omitted & \\
\hline Total $(n=66)$ & $51(61.44 \%)$ & $15(83.33 \%)$ & $3.14(0.84-11.7)$ & 0.09 \\
\hline
\end{tabular}

*Only $65.35 \%$ of the patients (66 patients) had bacterial growth in their sputum culture.

Table(15):Relation between the bacterial growth and patient's outcome $(\mathbf{n}=101) *$

Table (15) shows that there was a significant relation between poor outcome and isolation of Staphylococcus aureusand Pseudomonas aeruginosa from the sputum culture of the patients $(\mathrm{P}<0.0001$ and 0.002 respectively $)$.

\section{Discussion}

Our study included 101 patients (67 males and 34 females) diagnosed as AECOPD. $82.18 \%$ of the patients improved on treatment while $17.82 \%$ of them had poor outcome (i.e. $13.86 \%$ of the patients were referred to ICU and $3.96 \%$ of the patients died). According to our results, bacterial growth was recorded in 66 cases $(65.35 \%)$. In agreement with us, other study, in Upper Egypt, found significant bacterial growth in $77 \%$ of patients during $81 \%$ of exacerbations (their study included 156 patients who had 218 AECOPD during 18 months) (Hassan et al., 2016).Sethi and Murphy reviewed that at least $70-80 \%$ of the AECOPD are infectious in origin. Of these infections, 40 to $50 \%$ are caused by bacteria (Sethi and Murphy, 2008).
Our study found that the most commonly isolated bacteria in the sputum culture of the studied COPD patients were: Strept. pneumoniae, H. influenzae, P. aeruginosa and Staphy. aureus in $(14.85 \%, 13.87 \%, 10.89 \%$ and $8.9 \%$ of the patients respectively). The study of Hassan et al. recorded that the most commonly isolated bacterial strains during AECOPD were H. influenzae, Strept. pneumoniae, K. pneumoniae, and methicillin-resistant Staphylococcus aureus (MRSA) (18\%, $15 \%,, 14 \%$ and $11 \%$ ) (Hassan et al., 2016).Agmy et al., reported that the most predominant organisms in AECOPD were $H$. influnzae followed by Strept. Pneumonia and Maroxellacatarrhalis $(30 \%, 25 \%$ and $18 \%$ respectively) (Agmy et al., 2013). 
SOHAG MEDICAL JOURNAL

Vol. 23 No.1 Jan 2019

In this study, we found that the patients with poor outcome hadhigher mean age in comparison with the patients who improved; the mean age of the patient with poor outcome was 62.72 years in the other hand, the mean age among the patients with good prognosis was 59.84 years. Older patients were described to have worse clinical outcomes after acute exacerbation in many previous studies (Connors et al., 1996; Groenewegen et al., 2003, Roche et al., 2008 \&Flattet et al., 2017). The impact of age on survival may be explained by a gradual and natural decline in lung function, tendency to have multiple comorbidities, lower respiratory reserve and being more prone torespiratory muscle fatigue (Burrows et al., 1987\&Groenewegen et al., 2003). However, our study shows that there were no statistically significant relation between the outcome and the age $(\mathrm{P}=0.2)$, this result supports the findings of Gunen et al. that showed no significant relation between the age and outcome in patients with AECOPD (Gunen et al., 2005).

As regard the gender, our study found that male gender is a significant predictor of poor outcome ofAECOPD $(\mathrm{P}=0.04)$ in agreement with Singanayagam et al. (Singanayagam et al., 2013). In our community, female smoking is of less common than that of male gender, so most COPD in females is due to causes other than smoking. This may explain the milder form of the disease in females, as it was found that cigarette smokers have a higher prevalence of respiratory symptoms and lung function abnormalities, a greater annual rate of decline in $\mathrm{FEV}_{1}$ and a greater COPD mortality rate than nonsmokers (Rabe et al., 2007). Moreover, morbidity due to COPD was thought to be greater in men than women (Chapman, 2004). In contrast, other studies found no relation between
Acute Exacerbation of Chronic Obstructive

Esraa A. Saad.et al

the gender and clinical outcome in patients with AECOPD (Yousif et al., 2016 \&Elgazzar, 2018).

In our study, it was shown that poor outcome is significantly influenced by the higher frequencies of COPD exacerbation in the previous 12 months $(\geq 2$ exacerbation /year) $(\mathrm{P}=0.003)$. Gaude et al. found that frequent exacerbation was a predictor of poor outcome (death or treatment failure of AECOPD $) \quad(\mathrm{P}=0.001) \quad$ in their univariate logistic regression analysis (Gaude et al., 2015). Risk factors associated with this type of patient could be due to the rapid decline in lung function and respiratory bacterial or viral colonization. (Donaldson et al., 2002).

According to the present study, prior hospital admissions $\quad(\geq 2$ admission/year) and prior ICU admission, in the last year, also could predict poor outcome $(\mathrm{P}=0.004$ and 0.003 respectively). As regard prior hospitalization, we are in line with Gaude et al. who reported the prior hospitalization as a predictor of poor outcome, i.e. death or readmission, $(\mathrm{P}=$ 0.04) (Gaude et al., 2015). Elgazzar study also mentioned that the prior ICU admission was a predictor of poor outcome $(\mathrm{P}=0.042)$. These results could be explained as both history of previous hospitalization or previous ICU admission due to AECOPD reflect the severity of the underlying disease, poor pulmonary reserve, poor compliance to treatment and may be the resistance of infection (Elgazzar, 2018).

In our study, we also found that there was a significant relation between priorLTOT and poor outcome $(\mathrm{P}=$ 0.006). These results coincided with the previous studies that showed a significant relation between the LTOT and outcome in patients with AECOPD (Connors et al., 1996; Yohannes et al., 2005 \&Tsimogianni et al., 2009). 
SOHAG MEDICAL JOURNAL

Vol. 23 No.1 Jan 2019

LTOT reflects more severe stages of the disease that could be associated with corpulmonale, (which is a wellknown adverse prognostic factor) thus the relation between LTOT and poor outcome could be explained (Connors et al., 1996 \&Yohannes et al., 2005).

As regard the clinical parameters: altered consciousness and flapping tremor were predictors of outcome in our study $(\mathrm{P}=0.009 \quad \& \quad<0.0001$ respectively).Theyare considered as neurological manifestation of the hypercapnic encephalopathy which is caused by higher values of $\mathrm{PaCO}_{2}$ and respiratory acidosis. Roche et al also found that these factors were predictors of ICU admission or mortality (Roche et al., 2008).

With regard to vital signs, the present study also showed that the patients with poor outcome had higher mean rates of pulse and respiration, on admission, in comparison with patients with good outcome $(\mathrm{P}=0.02$ and $<0.0001$ in order). Tabak and collaegues considered tachycardia as a predictor of hospital mortality in patients with AECOPD (Tabak et al., 2009). Tachycardia may capture interactions between volume status, hypoxemia, and general distress. The risk of death or readmissions is increased in patients presenting severe tachypnea already noted across different studies, perhaps because respiratory rate is at an intersection ofmanypathophysiological processes, such as muscle dysfunction,respiratory failure and metabolic acidosis (Cretikos et al., 2008 \&Flattet et al., 2017).

Our results also demonstrates that fever had as a significant relation with the poor outcome $(\mathrm{P}=0.03)$. In agreement with us,fever was reported as a predictor of poor outcome, in ventilated COPD patients, in Elgazzar study which explained it byacting as a sign of systemic inflammatory
Acute Exacerbation of Chronic Obstructive

Esraa A. Saad.et al

response which may reflect the severity of the infection(Elgazzar, 2018).

Our study also demonstrates that pedal edema is a predictor sign for poor outcome $(\mathrm{P}=0.008)$, thus coincided withRoche et al. and Singanayagam et al. (Roche et al., 2008 \&Singanayagam et al., 2013). It could be explained as lower limb edema in COPD patient reflects the presence of right sided heart failure and more severe illness.

With respect to the pattern of comorbidities, in the present study, bronchiectasis andbronchiectatic changes in radiological assessmentwerefound to be significant predictors for poor outcome in AECOPD $\quad(\mathrm{P}=0.047) . \quad$ Previous research foundthat the patients with COPD and coexisting bronchiectasis have greater bronchial inflammation and greater chronic colonization of bronchial mucosa by a potentially pathogenic microorganism, this can lead to more frequent exacerbations with longer duration (Sethi and Murphy, 2001 \& Patel et al., 2002). In agreement with us, Du et al. found that comorbid bronchiectasis in COPD patients increased the risk of mortality (Du et al., 2016). In contrast to our results, Crisafulli et al.found that bronchiectasis had no effects on the clinical impact on hospital admission, the clinical presentation, the rate and the risk of short and long term mortality. Moreover, they found that in the patients with AECOPD, the prevalence of ICU admission in the patients with bronchiectasis was less than the prevalence of ICU admission in the patients without bronchiectasis (Crisafulli et al., 2018).

Corpulmonale and cardiomegaly weresignificant predictors for poor outcome in our results $(\mathrm{P}=0.005$ and 0.001 respectively). Previous studies considered corpulmonale to be a predictor of poor outcome in AECOPD 
SOHAG MEDICAL JOURNAL

Vol. 23 No.1 Jan 2019
Acute Exacerbation of Chronic Obstructive

Esraa A. Saad.et al
(Connors et al., 1996 and

Singanayagam et al., 2013).Cardiomegaly could be reflect presence of corpulmonale. It can be explained as they reflect the severity of the disease and could aggravate the acute illness.

In our study, diabetes mellitus appeared to be a significant factor in poor outcome prediction $(\mathrm{P}=0.008)$. In Baker et al., the higher blood glucose significantly predicted adverse clinical outcomes (death or prolonged hospital stay) in AECOPD (Baker et al., 2006). In acute illness, cytokines, hormones and hypoxia up regulate expression and membrane localization of glucose transporters in many cell types. Cellular glucose overload results in increased glucose metabolism, in turn increasing superoxide and peroxynitrite production which may impair mitochondrial activity (Van den Berghe, 2004).Hyperglycaemia could also cause adverse outcomes from AECOPD by predisposing to infection through systemic or local effects on host immunity or bacterial growth(Philips et al., 2003 \& Wood et al., 2004).

With respect to the arterial blood gas on admission: lower mean values of arterial blood pHat admission was a significantly associated with the poor outcome, according to our study $(\mathrm{P}=0.007)$.In contrast, Tsimogianniet al found that arterial blood $\mathrm{pH}$ was not related to mortality, and the author attributed this to the fact that the patients with significant respiratory acidosis were transferred to the intensive care unit and subsequently excluded from their study (Tsimogianni et al., 2009). In addition, lower values of $\mathrm{PaO}_{2}$ and $\mathrm{SaO}_{2} \%$ were found to be poor outcome predictors according to our study $(\mathrm{P}=0.003 \& 0.001)$.Gunen et al. also found that lower values of $\mathrm{PaO}_{2}$ are predictors of poor outcome andthey explained that because low $\mathrm{PaO}_{2}$ is a direct evidence for limited pulmonary reserve and increased ventilation/perfusion mismatch, thus, could reflects the severity of the underlying disease. These patients became less tolerant to alterations in their clinical condition, thus, showing a poorer prognosis (Gunen et al., 2005).Higher mean values of $\mathrm{PaCO}_{2}$ at hospital admission also was significantly associated with the poor outcome $(\mathrm{P}=\quad$ 0.01).As regard thesurvival in AECOPD,previous studies prescribed a worse prognosisin the case of high value of $\mathrm{PaCO}_{2}($ Groenewegen et al., 2003 \&Flattet et al., 2017). $\mathrm{PaCO}_{2}$ is a reflection of alveolar ventilation and is a reflection of the severity of the exacerbation.

As shown in the present study, poor outcome is related to leukocytosis $(\mathrm{P}=$ 0.008). In agreement with this, leukocytosis was found as a factor with a significant relation to poor outcome in hospitalized patients with AECOPD admitted to ICU, i.e. death(Ashmawi et al., 2017). Leukocytosis is an indicator of inflammation which reflects presence of infection in those patients so its relation with poor outcome in AECOPD could be explained. On the other hand, Elgazzar study foundthat leukocytosis were not related to outcome in AECOPD (Elgazzar et al., 2018).

Also, in the present study poor outcome was related to thrombocytopenia $\quad(\mathrm{P}=0.001)$.In agreement with that, thrombocytopenia was found to be a significant predictor of poor outcome (i.e. hospital mortality, need for ICU admission or mechanical ventilation, $\mathrm{P}=0.001,0.008$ and 0.001 respectively) in patients with AECOPD (Rahimi-Rad et al., 2015). Thrombocytopenia may reflect some pathophysiologic disturbances, including disseminated intravascular 
SOHAG MEDICAL JOURNAL

Vol. 23 No.1 Jan 2019

coagulation, sepsis, macrophage activation, vitamin deficiencies, druginduced toxicity orother unidentified factors (Moreau et al., 2007).

The present study showed that higher serum level of creatinine were associated with the risk of poor outcome $(\mathrm{P}=0.02)$. Fluttet et al., 2017 also found that impaired renal function is associated with poor outcome (i.e. mortality or readmission). A raised creatinine level in any acute medical condition may also represent an underlying poor hydration state prior to admission .

As regard elevated liver enzymes, we also observed that AECOPD patients with elevated serum levels of liver enzymes (ALT and AST) had higher risk of poor outcome $(\mathrm{P}=0.001)$. It could be explained by hypoxic hepatitis, due to arterial hypoxia and may be due to hepatic venous congestion in patients of severe decompensated corpulmonale(Henrion et al., 1999).

In our study, we also observed that hypoalbuminemiacould predict poor outcome $(\mathrm{P}=0.007)$. The importance of hypoalbuminemia as a predictor of poor outcome of AECOPD was consistent with results from other studies of AECOPD (Connors et al.,1996; Ai-Ping et al., 2005 \&Asiimwe et al., 2011). In contrast, Flattet et al. found no association between values of albumin and the clinical outcomes(Flattet et al., 2017). Low levels of this protein maybe due to combined effect of poor nutritional state in addition to the effect of the inflammation during AECOPD and it is also a good indicator for long-term health status in chronically ill patients. The suggested mechanisms for their roles in increased long-term mortality are respiratory muscle weakness, impaired gas exchange and impaired immune response (Connors et al., 1996\& Don and Kaysen, 2004).
Acute Exacerbation of Chronic Obstructive

Esraa A. Saad.et al

According to the echocardiography, severe pulmonary hypertension was a significant predictor of poor outcome in our results $(\mathrm{P}=0.03)$ in agreement with Hurdmanet al.(Hurdman et al., 2013), it could be explained of that they reflect the severity of the disease and could aggravate the acute illness.

Concerning lung function, our study showed that lower mean values of $\mathrm{FEV}_{1}$ and $\mathrm{FVC}$ had significant relation to poor outcome $(\mathrm{P}=0.01$ and 0.02 respectively) and the patient with very severe COPD (stage IV according to GOLD, 2018) had the worse outcome than the patients with less severity of the COPD $(\mathrm{P}=0.02)$. Such results have been reported in similar studies and are probably explained by the degree of ventilatory impairment and the higher risk of colonization by aggressive bacteria causing exacerbation (Miravitlles et al., 2000 \&Flattet et al., 2017).

Staph.aureusandP.aeruginosaisolation (in sputum culture) were predictors of poor outcome in our study $(\mathrm{P}<0.0001$ and 0.002 respectively). With respect to Staph.Aureus,Hassan et al. reported that it could be due to that all Staph.aureus strains in their COPD patients were MRSA (Hassan et al., 2016). This coincided with Borg et al. who found that the prevalence of MRSA in invasive isolates from blood cultures from nine hospitals in Egypt was 52\% (Borg et al., 2007).This high prevalence of MRSA in these studies should be an alarm for the increasing prevalence of MRSA among hospitalized patients in our locality.Previous studies agreed with the point that $\mathrm{P}$.aeruginosa isolation in patients with AECOPD is a risk for mortality (Renom et al., 2010\&Almagro et al., 2012). This microorganism is more frequent in advanced stages of the disease, and studies performed in outpatients with acute exacerbation showed an inverse 
SOHAG MEDICAL JOURNAL

Vol. 23 No.1 Jan 2019

relationship between P.aeruginosa and

pulmonary function. Thus, P.aeruginosa is a marker of severity in COPD, which explains the poor prognosis associated with it(GarciaVidal et al., 2009)However,Groenewegen et al. and Ko et al. reported that the presence of bacterial isolates did not influence the clinical outcomes, including the length of hospitalization and need for intensive care unit admission, and they did not discover the type of bacteria as a prognostic factor (Groenewegen et al., 2003 \&Ko et al. 2005).

\section{Conclusions}

- Bacterial isolation,in the sputum culture, was found in $65.35 \%$ of the patient with acute exacerbation of COPD .

- The most common bacterial strains, in sputum culture of patients with AECOPD were: Strept.pneumonia, H. influenza, P.aeruginosa, Staph.aureus .

- The significant factors in predicting poor outcome (ICU referral or death) in AECOPD were: male gender, prior LTOT, prior hospital admission ( $\geq 2$ admission/year) or prior ICU admission in the last year, frequent exacerbations, associated comorbidities

(bronchiectasis,corpulmonale and DM), consciousness alteration, tachycardia, tachypnea, fever, flapping tremor, pedal edema, arterial blood gas parameters (higher mean values of $\mathrm{PaCO}_{2}$ and lower mean values of $\mathrm{pH}$, $\mathrm{PaO}_{2} \& \mathrm{SaO}_{2}$ ), leukocytosis, thrombocytopenia, elevated serum creatinine,elevated liver enzymes(ALT\& AST), hypoalbuminemia, presence of cardiomegaly or bronchiectatic changes as radiological findings, severe pulmonary artery hypertension according to echocardiography, lower mean values of $\mathrm{FEV}_{1}$ and $\mathrm{FVC}$, and
Acute Exacerbation of Chronic Obstructive Esraa A. Saad.et al

Staph.aureusor P.aeruginosaisolation in sputum culture of AECOPD patients.

\section{References}

1. Agmy G, Mohamed S, Gad Y, Farghally E, Mohammedin $\mathrm{H}$ and Rashed H (2013) Bacterial profile, antibiotic sensitivity and resistance of lower respiratory tract infections in Upper Egypt. Mediterr J Hematol Infect Dis.; 5: e2013056.

2. Ai-Ping C, Lee K and Lim T (2005) Inhospital and 5-year mortality of patients treated in the ICU for acute exacerbation of COPD: a retrospective study. Chest; 128: 518-524.

3. Almagro P., Cabrera F. J., Diez J Boixeda R, Alonso Ortiz MB, Murio C and Soriano JB (2012) Comorbidities and short-termprognosis in patients hospitalized for acute exacerbation of COPD: the EPOC en servicios de medicinainterna (ESMI) study. Chest; 142(5):1126-1133.)

4. Anthonisen N, Manfreda J, Warren CP,Hershfield ES, Harding GK and Nelson NA (1987) Antibiotic therapy in exacerbations of chronic obstructive pulmonary disease. Ann Intern Med.; 106:196-204.

5. Ashmawi S,Riad N \&Saeed M. (2017) Assessment of serum Retinol-Binding Protein-4 Levels in patients with acute exacerbation of chronic obstructive disease at intensive care unit. Egyptian Journal of Chest Diseases \& Tuberculosis; 66: 739-743

6. Asiimwe A, Brims F, Andrews N, Prytherch, D, Higgins, B, Kilburn, S \&Chauhan, A (2011) Routine laboratory tests can predict in-hospital mortality in acute exacerbations of COPD. Lung; 189(3): 225-232.

7. Baker E, Janaway C, Philips B, Brennan A, Baines D Wood DM and Jones PW (2006) Hyperglycaemia is associated with poor outcomes in patients admitted to hospital with acute exacerbations of chronic obstructive pulmonary disease. Thorax; 61(4): 284-289.

8. Borg M, De Kraker M, Scicluna E, Van de Sande-Bruinsma N, Tiemersma E, Monen J and Grundmann H (2007) 
SOHAG MEDICAL JOURNAL

Vol. 23 No.1 Jan 2019

ARMed Project Members and

Collaborators: Prevalence of

methicillin-resistant Staphylococcus

aureus (MRSA) in invasive isolates

from southern and eastern

Mediterranean countries. J

AntimicrobChemother ; 60:1310-1315.

9. Burrows B, Bloom J, Traver G and Cline M (1987) The course and prognosis of different forms of chronic airways obstruction in a sample from the general population. N Engl J Med.; 317(21): 1309-1314.

10. Chapman K. (2004) Chronic obstructive pulmonary disease: are women more susceptible than men?.Clin Chest Med.; 25 (2): 331341.

11. Chow AW, Hall CB, Klein JO, Kammer RB, Meyer RD and Remington JS (1992) Evaluation of new anti-infective drugs for the treatment of respiratory tract infections. Clin Infect Dis.;15:S62-S88.

12. Connors A., Dawson N., Thomas C., Harrell F., Desbiens N., Fulkerson WJ, et al. (1996) Outcomes following acute exacerbation of severe chronic obstructive lung disease. The SUPPORT investigators (Study to Understand Prognoses and Preferences for Outcomes and Risks of Treatments). Am J RespirCrit Care Med.;154:959-967.

13. Cretikos M, Bellomo R, Hillman $\mathrm{K}$, Chen J, Finfer $\mathrm{S}$ and Flabouris A (2008) Respiratory rate: the neglected vital sign. Med J Aust.; 188(11): 657659.

14. Crisafulli E., Guerrero M., Ielpo A., Ceccato A., Huerta A., Gabarrús A., Soler N., Chetta A. and Torres A. (2018) Impact of bronchiectasis on outcomes of hospitalized patients with acute exacerbation of chronic obstructive pulmonary disease: A propensity matched analysis. Scientific Reports; volume 8,

Article number: 9236.

15. Don BR. and Kaysen G. 2004 Serum albumin: relationship to inflammation and nutrition.Semin Dial.;17(6):432437.

16. Donaldson G., Seemungal T., Bhowmik A. and Wedzicha J.
Acute Exacerbation of Chronic Obstructive Esraa A. Saad.et al

(2002) Relationship between exacerbation frequency and lung function decline in chronic obstructive pulmonary disease. Thorax; 57: $847-$ 852.

17. Du et al., 2016: Du Q., Jin J., Liu X. and Sun Y. (2016) Bronchiectasis as a comorbidity of chronic obstructive pulmonary disease: a systematic review and meta-analysis. PLoS One;11(3):e0150532.

18. Elgazzar A. (2018) Clinical phenotype as a predictor of outcome in mechanically ventilated chronic obstructive pulmonary disease patients. Egypt J Bronchol.; 12: 180-186.

19. Elkorashy R and Elsherif R.H (2014) Gram negative organisms as a cause of acute exacerbation of COPD. Egyptian $\mathrm{J}$ of Chest Disease and Tuberculosis; pp: 345-349.

20. Flattet $Y$, Garin N, Serratrice J, Perrier A, Stirnemann J and Carballo S (2017) Determining prognosis in acute exacerbation of COPD. International Journal of COPD; $12: 467-475$.

21. Garcia-Vidal C, Almagro P, Romaní V, Rodríguez-Carballeira M, Cuchi E, Canales L, et al. (2009) Pseudomonas aeruginosa in patients hospitalised for COPD exacerbation: a prospective study.EurRespir J.;34(5):1072-8.

22. Gaude G., Rajes h B., Chaudhury A., and Hattiholi J. (2015) Outcomes associated with acute exacerbations of chronic obstructive pulmonary disorder requiring hospitalization. Lung India; 32(5): 465-472.

23. Global Initiative for Chronic Obstructive Lung Disease(GOLD): Global strategy for diagnosis, management, and prevention of COPD (2018).

24. Groenewegen K., Schols A. and Wouters E. (2003) Mortality and mortality-related factors after hospitalization for acute exacerbation of COPD. Chest; 124(2): 459-467.

25. Groenewegen, K.H. and Wouters, E.F. Bacterial infections in patients requiring admission for an acute exacerbation of COPD; a 1-year prospective study. Respir Med. 2003; 97: 770-777. 
SOHAG MEDICAL JOURNAL

Vol. 23 No.1 Jan 2019

26. Gunen $\mathrm{H}$, Hacievliyagil S, Kosar F, Mutlu L, Gulbas G, Pehlivan E et al. (2005) Factors affecting survival of hospitalized patients with COPD. EurRespir J.; 26: 234-241.

27. Hassan A., Mohamed S., Mohamed M. and El-Mokhtar M. (2016) Acute exacerbations of chronic obstructive pulmonary disease: Etiological bacterial pathogens and antibiotic resistance in Upper Egypt. Egypt J Bronchol.; 10: 283-290.

28. Henrion $J$, Minette $P$, Colin L, Schapira M, Delannoy A and Heller F (1999) Hypoxic Hepatitis Caused by Acute Exacerbation of Chronic Respiratory Failure: A CaseControlled, Hemodynamic Study of 17 Consecutive Cases. Hepatology; 29: 427-433.

29. Hurdman J, Condliffe R, Elliot CA, Swift A, Rajaram S, Davies C, et al. (2013) Pulmonary hypertension in COPD: results from the ASPIRE registry. European Respiratory J., 41 (6) $1292-1301$.

30. Ko FW, Ng TK, Li TS, Fok JP, Chan $\mathrm{MC}, \mathrm{Wu}$ AK and Hui DS. (2005) Sputum bacteriology in patients with acute exacerbations of COPD in Hong Kong. Respir. Med.; 99: 454-460.

31. Lopez A., Shibuya K., Rao C., Mathers C., Hansell A., Held L., et al (2006) Chronic obstructive pulmonary disease: current burden and future projections. EurRespir J.; 27(2):397-412.

32. Mantero M., Rogliani P, Di Pasquale M, Polverino E., Crisafulli E,Guerrero $M$ et al. (2017) Acute exacerbations of COPD: risk factors for failure and relapse. Int J Chron Obstruct Pulmon Dis.; 12: 2687-2693.

33. Miravitlles M, Guerrero $\mathrm{T}$, Mayordomo C, Sanchez-Agudo L, Nicolau F and Segú JL (2000) Factors associated with increased risk of exacerbation and hospital admission in a cohort of ambulatory COPD patients: a multiple logistic regression analysis. The EOLO Study Group. Respiration; 67(5): 495501.

34. Moreau D, Timsit JF, Vesin A, Garrouste-Orgeas M, de Lassence A, Zahar JR, et al. Platelet count decline: an early prognostic marker in
Acute Exacerbation of Chronic Obstructive Esraa A. Saad.et al

critically ill patients with prolonged ICU stays. Chest. 2007;131(6):17351741.

35. Murray P., Baron E., Jorgensen J., Pfaller M. and Yolken R. (2003) Manual of clinical microbiology, 8th ed. Washington, DC,USA: ASM Press.

36. Patel I., Seemungal T., Wilks M., Lloyd-Owen S., Donaldson G and Wedzicha J (2002) Relationship between bacterial colonization and the frequency, character, and severity of COPD exacerbations. Thorax;57(9):759-764.

37. Philips $\mathrm{B}$, Meguer $\mathrm{J}$, Redman $\mathrm{J}$ and Baker E (2003) Factors determining the appearance of glucose in upper and lower respiratory tract secretions. Intensive Care Med.; 29(12): 22042210.

38. Rabe K, Hurd S, Anzueto A,Barnes PJ, Buist SA, Calverley P et al., (2007) Global strategy for the diagnosis, management, and prevention of chronic obstructive pulmonary disease: GOLD executive summary. Am J RespirCrit Care Med.; 176: 532-555.

39. Rahimi-Rad MH, Soltani S, Rabieepour $\mathrm{M}$ and Rahimirad $\mathrm{S}$ (2015) Thrombocytopenia as a marker of outcome in patients with acute exacerbation of chronic obstructive pulmonary disease. PneumonolAlergol Pol.;83(5):348-51.

40. Renom F., Yáñez A., Garau M., Rubí M., Centeno MJ.Gorriz MT., et al. (2010) Prognosis of COPD patients requiring frequent hospitalization: Role of airway infection. Respiratory Medicine; 104 (6): 840-848.

41. Roche N., Zureik M., Soussan D., Neukirch F. and Perrotin D. (2008) Urgence BPCO (COPD Emergency) Scientific Committee. Predictors of outcomes in COPD exacerbation cases presenting to the emergency department. EurRespir J.; 32(4): 953961.

42. Seemungal T., Donaldson G., Bhowmik A., Jeffries D. and Wedzicha J. (2000) Time course and recovery of exacerbations in patients with chronic obstructive pulmonary disease. Am J RespirCrit Care Med.;161:1608-1613. 
SOHAG MEDICAL JOURNAL

Vol. 23 No.1 Jan 2019

43. Sethi S and Murphy T (2001) Bacterial infection in chronic obstructive pulmonary disease in 2000: a state of the art review. ClinMicrobiol Rev.;14:336-363.

44. Sethi $S$ and Murphy $T$ (2008) Infection in the pathogenesis and course of chronic obstructive pulmonary disease. N Engl J Med.;359(22):2355-65.

45. Singanayagam A., Schembri S. and Chalmers J. (2013) Predictors of mortality in hospitalized adults with acute exacerbation of chronic obstructive pulmonary disease. Ann Am Thorac Soc.; 10(2): 81-89.

46. Tabak Y., Sun X., Johannes R., Gupta V. and Shorr A. (2009) Mortality and need for mechanical ventilation in acute exacerbations of chronic obstructive pulmonary disease: development and validation of a simple risk score. Arch Intern Med.; 169(17): 1595-1602.

47. Tsimogianni A, Papiris S, Stathopoulos G, Manali E, Roussos C and Kotanidou A (2009) Predictors of Outcome After
Acute Exacerbation of Chronic Obstructive

Esraa A. Saad.et al

Exacerbation of Chronic Obstructive Pulmonary Disease. Society of General Internal Medicine; 24(9): 1043-1048.

48. Van den Berghe G (2004) How does blood glucose control with insulin save lives in intensive care?. J Clin Invest.; 114(9): 1187-1195.

49. Wood D, Brennan A, Philips B and Baker E (2004) Effect of hyperglycaemia on glucose concentration of human nasal secretions. ClinSci (Lond); 106(5): 527-533.

50. Yohannes A., Baldwin R. and Connolly M. (2005) Predictors of 1-year mortality in patients discharged from hospital following acute exacerbation of chronic obstructive pulmonary disease. Age Ageing; 34 (5): 491-496.

51. Yousif M. and El Wahsh R. (2016) Predicting in-hospital mortality in acute exacerbation of COPD: Is there a golden score? Egyptian Journal of Chest Diseases and Tuberculosis; 65: 579-584. 